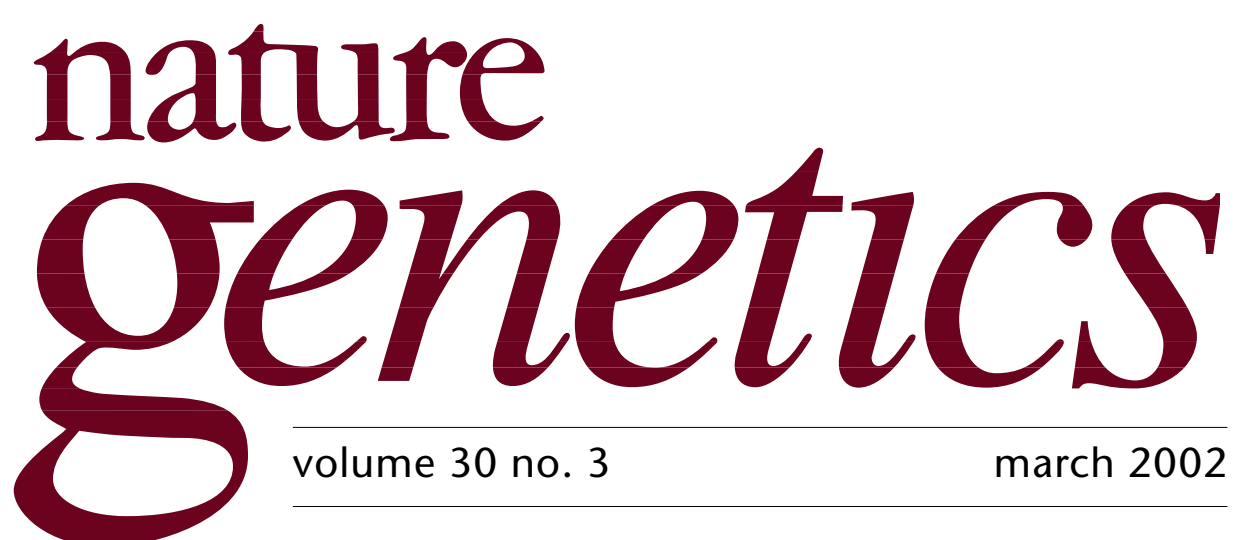

\title{
Share and share alike?
}

The advancement of science depends on the sharing of information. As the validation of a result depends on its replication, genetics journals, including this one, make the sharing of materials and methods a condition of publication. And yet a recent survey ${ }^{1}$ of 1,240 US academic geneticists carried out by Eric Campbell, David Blumenthal (Institute for Health Policy, Massachusetts General Hospital) and colleagues indicates that $28 \%$ of respondents have been unable to confirm published research because they were denied access to the necessary information by the authors of the original work.

This statistic deserves consideration by researchers, editors, repositories, research bodies and funding agencies, including industrial sponsors. Additional measurements made by Campbell et al. ${ }^{1}$ are helpful. They have recorded the reasons cited by geneticists for withholding post-publication information, data or materials (see figure). They also collected and analyzed responses from life scientists who are not geneticists for comparison.

The most frequently cited reason for withholding post-publication information, data or materials is the effort required to produce the materials and information. Consistent with this result are that requests for biomaterials are more likely to be declined than requests for information, and those who receive more requests are significantly more likely to decline a request. The authors propose two possible contributing factors: the scarcity of precious materials and the demanding and complex nature of material transfer agreements (MTAs).

The experience of the mouse geneticist Tak Mak (Univ. Toronto and Amgen Research Institute) illustrates difficulties that some researchers face when distributing knockout mice. Some of his requests to submit mice to The Jackson Laboratory have been declined, owing to limited capacity. He receives on average three to four requests for a knockout mouse or mutant tissues each day. For each mouse or tissue supplied from his laboratory, he must arrange an MTA. This involves correspondence with a lawyer at Amgen, a lawyer at the institute of the person making the request, and the veterinarians at his animal facility and the receiving facility. If the mouse is to be exported from Canada, correspondence with the agriculture department of the recipient's country is also necessary. Sometimes, there are biological limitations-some knockouts, such as Apaf1 ${ }^{-/}$or $\mathrm{Pten}^{-/}$, are embryonic lethals or difficult to breed, and breeding heterozygotes involves the production of more mice, which costs more money. Mak estimates that he is able to meet $15-20 \%$ of the requests that he receives, and says "every year, we are more years behind in fulfilling requests."

As Mak must seek an MTA from Amgen for each mouse that is requested, his situation represents one end of the spectrum with respect to quantities of paperwork. 


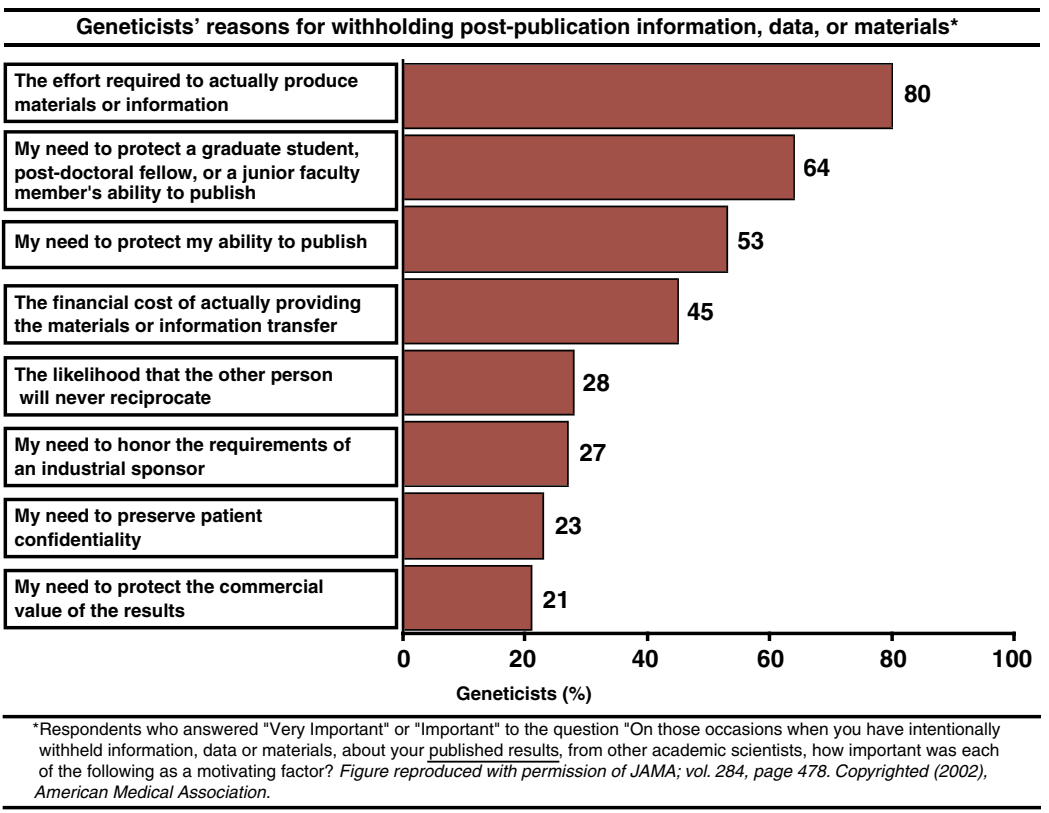

However, it is one that will be familiar to an increasing number of academic geneticists, given the rise in research relationships between genetics firms and academic institutes ${ }^{2}$. Campbell and colleagues reported a trend of more denying among geneticists receiving industrial support $(P=0.07)$, and significantly more denials of requests among geneticists engaged in commercial activities $(P=0.03)$.

Commercial restrictions aside, the ability of mouse repositories to receive and store the knockout and mutant mice-whose numbers continue to grow-is paramount to ensuring their dissemination. And so the inability of The Jackson Laboratory to accept more than 100 mice each year is of concern. Barbara Knowles, of The Jackson Laboratories, says that the cost of providing robust customer service support, in addition to the handling of mice and, where necessary, MTA arrangements, is the limiting factor. It is for this reason that the Laboratory is now engaged in strategic planning talks, with a view to increasing its stocks through the use of frozen embryos and cryopreserved sperm (see page 255 for a related Communication). Improved manipulation of mutant oocytes and recent advances in intracytoplasmic sperm injection are also expected to improve productivity.

Another potential means of lowering the 'effort' threshold is the inclusion of funds dedicated to this purpose in grant or supplementary funding applications, as recently suggested ${ }^{3}$ by Wendy Baldwin (the deputy director of the extramural division of the National Institutes of Health).

Campbell and Neil Holtzman (of Johns Hopkins Univ. and an author of the sur$v^{1} y^{1}$ ), however, have reservations about the extent to which additional funding would help. The second and third most frequently cited reasons for declining a request are to do with the ability to publish — which discloses, says Holtzman, "a fundamental problem in the minds of some researchers about data sharing that is exacerbated by universities encouraging entrepreneurship". He says that citing effort as an obstacle may be an "easy way out".

It is certainly plausible that some researchers find it more of an 'effort' to send reagents to a perceived competitor than to someone who is less likely to pub-

Campbell, E.G. et al. JAMA 287, 473-480 (2002).

2. Blumenthal, D., Causino, N. \& Campbell, E.G. Nature Genet. 16, 104-108 (1997).

3. Stokstad, E. Science 295, 599 (2002) lish competitively. Such an impulse is understandable, but must be resisted. Willfully withholding available materials and information, post publication, from those who would make use of them compromises the process for ensuring the integrity of the scientific record and retards scientific advance. 